\title{
BSR ABSTRACT (447/450words)
}

Title: Sex-related differences in muscle co-activation in individuals with knee osteoarthritis SEX-RELATED DIFFERENCES IN MUSCLE CO-ACTIVATION IN INDIVIDUALS WITH KNEE OSTEOARTHRITIS

Authors: Stephanie L Smith, Jim Woodburn, Martijn Steultjens

Stephanie.Smith@gcu.ac.uk

Jim.Woodburn@gcu.ac.uk

Martijn.Steultiens@gcu.ac.uk

Institutions: Centre for Living, Institute for Applied Health Research, School of Health and Life Sciences, Glasgow Caledonian University, Glasgow, G4 OBA

Conflicts of Interest: The authors have no conflicts of interest to declare for this work. Funding: None

Background: Sex-related differences in muscle function have been well established in healthy individuals. In individuals with knee osteoarthritis (KOA) impairments in muscle function such as muscle weakness and high muscle co-activation have also been demonstrated. Muscle dysfunction has been shown to be a strong contributor to poor physical function and low health-related quality of life in patients with KOA. The purpose of this study was, therefore, to analyse sex and osteoarthritis-related differences in muscle function, to establish to what extent both sex and disease status contribute to muscle dysfunction.

Methods: Muscle co-activation was assessed in 77 symptomatic KOA participants (62.5 $\pm 8.1 \mathrm{yrs}$; 48/29 women/men) and 18 age-matched asymptomatic controls (62.5 $\pm 10.4 \mathrm{yrs} ; 9 / 9$ women/men), using electromyography (EMG) during a series of walking, stair ascent and descent and sit-to-walk activities. EMG was recorded from 7 sites medial/lateral gastrocnemius, biceps femoris, semitendinosus, vastus lateralis/medialis and normalised to maximal voluntary contraction. Normalised EMG was used to calculate hamstrings-quadriceps and medial-lateral muscle coactivation as (antagonist/agonist)*(antagonist+agonist). The stance phase of walking was split into pre-stance (150ms prior to initial contact), loading (0-15\% of stance), early-stance (15-40\%), midstance $(40-60 \%)$, late-stance $(60-100 \%)$ and overall-stance (0-100\%). Stairs negotiation was also split into transition (stance phase on the floor) and continuous (stance phase on the second step of the staircase). All participants provided written informed consent and the study was approved by Research Ethics committees (HLS12/86, 13/ws/0146). Independent samples T-tests were performed to assess the differences between KOA and controls. Linear regressions were performed to investigate the relationship between muscle function, sex and disease status, and Bonferroni corrected for multiple comparisons.

Results: Individuals with KOA were weaker than controls $(P<0.007)$. Overall there were very few differences in muscle co-activation between KOA and controls. Women were weaker than men $(P \leq 0.002)$ and had higher hamstrings-quadriceps and medial-lateral muscle co-activation across all activities of daily living. In multiple regression analyses sex and muscle weakness, but not age or disease status, predicted high muscle co-activation. 
Conclusions: High muscle co-activation was associated with female sex and muscle weakness regardless of disease status and age. It has previously been suggested that muscle co-activation acts as a compensatory mechanism for muscle weakness, accommodating for the diminished force generating capabilities to maintain a certain level of function and movement activation patterns. This suggests that muscle weakness may be the main contributing factor for high muscle coactivation which is thought to increase joint loads with detrimental effects on cartilage and joint integrity. This may explain high muscle co-activation in women with muscle weakness and increased risk of incidence and progression of KOA in women. 